\title{
Pembrolizumab plus axitinib: a new treatment option for patients with metastatic renal cell carcinoma
}

\author{
Veronica Mollica, Vincenzo Di Nunno, Francesco Massari \\ Division of Oncology, S. Orsola-Malpighi Hospital, Bologna, Italy \\ Correspondence to: Francesco Massari, MD. Division of Oncology, S. Orsola-Malpighi Hospital, Via Albertoni n 15, 40138 Bologna, Italy. \\ Email: fmassari79@gmail.com. \\ Provenance: This is an invited article commissioned by the Guest Section Editor Kaiping Zhang (PhD, AME College, AME Group, Hangzhou, China). \\ Comment on: Rini BI, Plimack ER, Stus V, et al. Pembrolizumab plus Axitinib versus Sunitinib for Advanced Renal-Cell Carcinoma. N Engl J Med \\ 2019;380:1116-27.
}

Submitted Apr 14, 2019. Accepted for publication Apr 22, 2019.

doi: 10.21037/cco.2019.04.05

View this article at: http://dx.doi.org/10.21037/cco.2019.04.05

The treatment scenario for patients with metastatic renal cell carcinoma (mRCC) is witnessing its third revolution: the first was the advent of anti-angiogenic targeted therapies, consisting in tyrosine-kinase inhibitors (TKIs) targeting vascular endothelial growth factor (VEGF); the second was the introduction of immunotherapy, consisting in immune checkpoint inhibitors (ICI), like antiprogrammed death 1 (PD-1) receptor or anti-programmed death ligand 1 (PD-L1); the third and current revolution is the advent of ICI and TKIs combination.

The biological rationale of this combination lies on the known effect of anti-angiogenic therapies of normalizing tumor vascularization and activating endothelial cells, leading to a higher infiltration of intra-tumoral T-cells. This could result in a synergistic effect of the blockade of PD-1/PD-L1 and VEGFR, leading to an enhanced effect of both therapies $(1,2)$.

The results of two studies investigating combination of ICI and anti-angiogenic therapy compared to sunitinib have been recently published, proving the benefit of the combination over the anti-angiogenic therapy alone: Keynote-426 (3), investigating the combination of pembrolizumab, an anti-PD1, plus axitinib, a VEGFR inhibitor, and Javelin-Renal 101 (4), investigating the combination of avelumab, an anti-PD-L1, plus axitinib.

The primary endpoints of the Keynote-426 were overall survival and progression-free survival in the intention-to-treat population. After a median follow-up of 12.8 months, pembrolizumab plus axitinib resulted in better overall survival (HR 0.53, $\mathrm{P}<0.0001)$, median progression free survival (15.1 months in the combination arm versus 11.1 months in the sunitinib arm, HR 0.69, $\mathrm{P}<0.001$ ) and objective response rate $(59.3 \%$ in the combination arm versus $35.7 \%$ in the sunitinib arm), with a higher percentage of complete response $(5.8 \%$ in the combination arm versus $1.9 \%$ in the sunitinib arm). The percentage of complete response with pembrolizumab plus axitinib were also higher than those obtained with avelumab plus axitinib, both in the PD-L1 positive population $(4.4 \%)$ and the overall population (3.4\%). The only combination to achieve a better rate of complete response is nivolumab plus ipilimumab in the CheckMate-214 (9\% in the intermediate and poor risk population) (5). Nonetheless, it should be underlined that in the Keynote-426 a higher percentage of favorable risk patients than in the Javelin-Renal 101 was enrolled (31.9\% and $21.7 \%$ in the overall population, respectively).

Pembrolizumab plus axitinib proved its efficacy in all the risk categories according to International Metastatic Renal Cell Carcinoma Database Consortium (IMDC) criteria (6), differently to nivolumab plus ipilimumab combination that has been approved only for intermediate and poor risk categories, and independently to PD-L1 expression. Differently, the study Javelin-Renal 101 restricted the evaluation of the primary endpoints PFS and OS to the $63.2 \%$ of patients with PD-L1 positivity, thus narrowing the field in which this combination could be used; moreover, it should be underlined that the method of evaluation of PD-L1 is not standardized in RCC thus different methods were used in each trial.

The results of these three combination trials are adding new standards of care in I line treatment of mRCC and will 
probably be incorporated in future guidelines. So, what is left to other treatment strategies such as TKIs monotherapy? And when should we use the ICI plus TKI combination or the ICI plus ICI combination?

One of the first questions that arises is if it is better to start with a combination directed against both targets (immune system and angiogenesis) upfront or if it could be beneficial to use it sequentially. In the pembrolizumab plus axitinib arm, $50 \%$ of patients that discontinued therapy started a second line of treatment, most of which with an anti-angiogenic therapy (44.3\%). Differently, in the sunitinib arm, $60.7 \%$ patients received a subsequent therapy, mainly balanced between anti-PD1/PD-L1 (37.6\%) and anti-angiogenic therapy (35.5\%). It should be pondered if the combination really adds some benefit in term of survival outcomes compared to a sequential treatment of nivolumab plus ipilimumab followed by an anti-angiogenic treatment or only useless toxicities.

In fact, the outstanding results of pembrolizumab plus axitinib have been achieved at a cost in term of toxicities that should be carefully considered. Pembrolizumab plus axitinib combination resulted in a higher percentage of all treatment discontinuation compared with avelumab plus axitinib (10.7\% and $7.6 \%$, respectively), while a lower percentage compared to nivolumab plus ipilimumab (22\%). Furthermore, $20.3 \%$ of patients had to reduce the dose of axitinib compared with $30.1 \%$ of patients that underwent sunitinib dose reduction. In terms of possible immunemediated events, $51.3 \%$ of patients in the pembrolizumab plus axitinib arm experienced any event of any grade compared with $38.2 \%$ in the avelumab plus axitinib arm of the Javelin-Renal 101.

Furthermore, it should be underlined that the majority of patients in the pembrolizumab plus axitinib arm had two or more metastatic sites at baseline (72.9\%), thus it could be hypothesized that patients with a higher burden of disease could benefit more from a combined therapeutic approach upfront.

Moreover, it should be noted that CheckMate-214 is the trial with the longest median follow-up of the three analyzed, with a median follow-up of 25.2 months compared to 12.8 months of the Keynote-426 and 11.6 months of the Javelin-Renal 101. Therefore, we should compare the results of these trials with caution awaiting for more mature results of the other two studies.

The treatment scenario of $\mathrm{mRCC}$ is about to be revolutionized: for favorable risks patients remains the option of single therapy with TKIs, consisting mainly of sunitinib, pazopanib, tivozanib or cabozantinib, but the combination of ICI plus TKI could be another standard of care, so it will be interesting to find which patients could benefit from one treatment or the other. For the intermediate and poor risk categories, there is no clear winner between nivolumab plus ipilimumab and ICI plus axitinib. The decision in this subset should be made according to patients' characteristics, contraindication to each therapy, like autoimmune disease or chronic use of corticosteroids for ICI, toxicities and the response needed in terms of disease control or achievement of complete or partial response. On this line, ICIs should be carefully used in patients with a need for a quick tumor shrinkage because of the risk of hyperprogression associated with these treatments.

So, is the IMDC criteria really enough to depict the complexity and the variety of mRCC patients' characteristics? In addition to the clinical and laboratoristic parameters currently validated to stratify patients, many biological and genetic features of the disease are being studied to deepen our knowledge of this tumor. For the localized setting, Rini et al. developed a 16-gene assay that correlates to clinical outcome in patients undergoing curative nephrectomy, providing a more accurate assessment of risk of recurrence, but this approach is not currently transferrable to clinical practice and it is not valid for the metastatic setting (7). Furthermore, pre-specified analyses of the IMmotion-151 trial, that compared atezolizumab plus bevacizumab versus sunitinib in previously untreated patients with mRCC, showed how two main gene signatures could be identified: angiogenesis and T-effector gene signature (8). Sunitinib resulted to be more effective in patients with angiogenesis high gene signature, while atezolizumab plus bevacizumab improved PFS versus sunitinib in patients with T-effector high and angiogenesis low gene signature. The molecular approach to stratify patients could be an answer to an unmet clinical need, especially in this historical moment in which different therapeutic approaches have been demonstrated to be effective, but its costs deeply reduce its clinical use.

Expanding the treatment strategies at our disposal leads directly to the need for predictive and prognostic biomarkers to better stratify our patients and to guide our clinical decision to find which patients should be treated with a combination approach and which could be selected for a single-agent treatment sparing some toxicities.

The efficacy and toxicities outcomes of ICI plus axitinib, sunitinib and nivolumab plus ipilimumab are reported in Tables 1 and 2, respectively. 


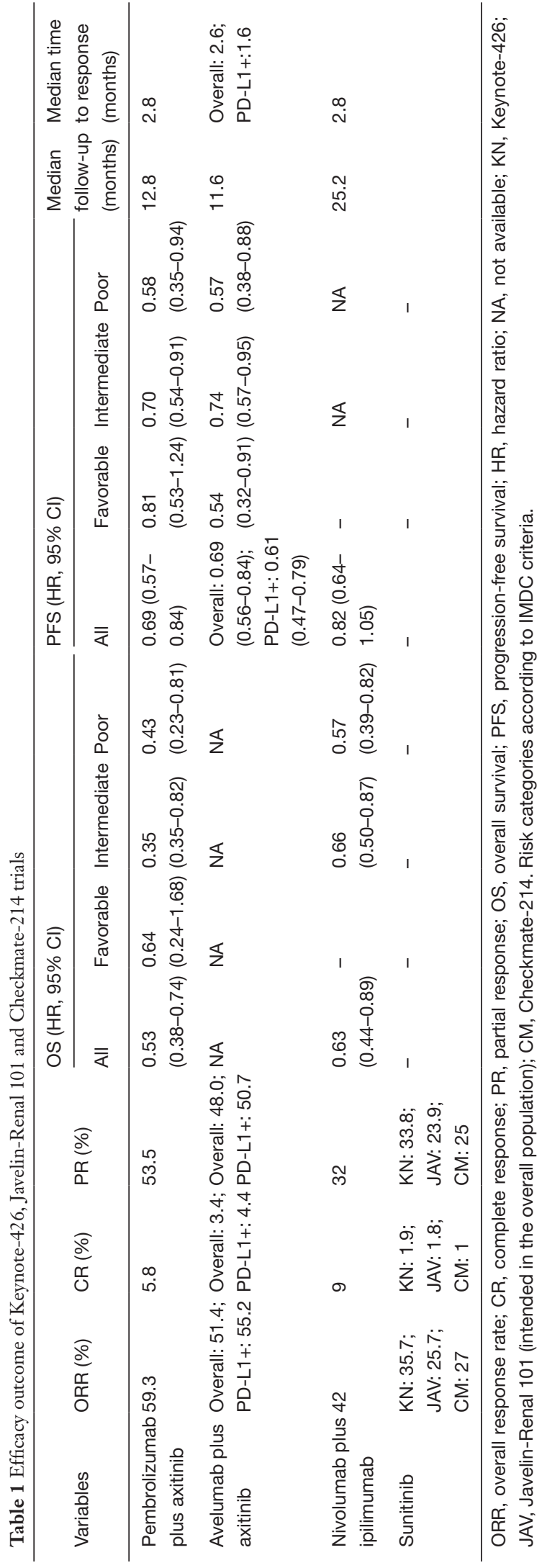

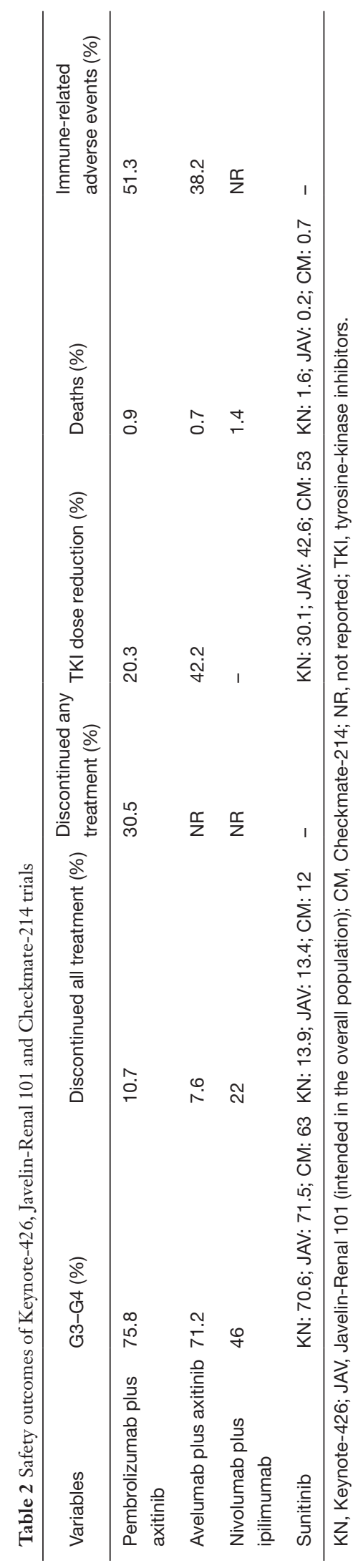




\section{Acknowledgments}

None.

\section{Footnote}

Conflicts of Interest: The authors have no conflicts of interest to declare.

\section{References}

1. Turajlic S, Swanton C, Boshoff C. Kidney cancer: The next decade. J Exp Med 2018;215:2477-9.

2. Yasuda S, Sho M, Yamato I, et al. Simultaneous blockade of programmed death 1 and vascular endothelial growth factor receptor 2 (VEGFR2) induces synergistic antitumour effect in vivo. Clin Exp Immunol 2013;172:500-6.

3. Rini BI, Plimack ER, Stus V, et al. Pembrolizumab plus Axitinib versus Sunitinib for Advanced Renal-Cell Carcinoma. N Engl J Med 2019;380:1116-27.

Cite this article as: Mollica V, Di Nunno V, Massari F. Pembrolizumab plus axitinib: a new treatment option for patients with metastatic renal cell carcinoma. Chin Clin Oncol 2019;8(Suppl 1):S21. doi: 10.21037/cco.2019.04.05
4. Motzer RJ, Penkov K, Haanen J, et al. Avelumab plus Axitinib versus Sunitinib for Advanced Renal-Cell Carcinoma. N Engl J Med 2019;380:1103-15.

5. Motzer RJ, Tannir NM, McDermott DF, et al. Nivolumab plus Ipilimumab versus Sunitinib in Advanced Renal-Cell Carcinoma. N Engl J Med 2018;378:1277-90.

6. Heng DY, Xie W, Regan MM, et al. Prognostic factors for overall survival in patients with metastatic renal cell carcinoma treated with vascular endothelial growth factortargeted agents: results from a large, multicenter study. J Clin Oncol 2009;27:5794-9.

7. Rini B, Goddard A, Knezevic D, et al. A 16-gene assay to predict recurrence after surgery in localised renal cell carcinoma: development and validation studies. Lancet Oncol 2015;16:676-85.

8. Rini BI, Huseni M, Atkins MB, et al. Molecular correlates differentiate response to atezolizumab (atezo) + bevacizumab (bev) vs sunitinib (sun): results from a Phase III study (IMmotion151) in untreated metastatic renal cell carcinoma (mRCC). ESMO 2018, abstract LBA 31. 